Corrigendum

\title{
Corrigendum to "Beta-Thalassemia Intermedia: A Single Thalassemia Center Experience from Northeastern Iraq"
}

\author{
Shaema Salih Amin $\left(\mathbb{D},{ }^{1}\right.$ Sana Dlawar Jalal $\mathbb{D}^{2}{ }^{2}$ Kosar Muhammed Ali, ${ }^{3}$ \\ Ali Ibrahim Mohammed, ${ }^{2}$ Luqman Khalid Rasool, ${ }^{4}$ and Tara Jamel Osman ${ }^{4}$ \\ ${ }^{1}$ Sulaymaniyah Directorate of Health, Hewa Oncology Hospital, Sulaymaniyah, Iraq \\ ${ }^{2}$ Department of Pathology, College of Medicine, University of Sulaymaniyah, Sulaymaniyah, Iraq \\ ${ }^{3}$ Department of Medicine, College of Medicine, University of Sulaymaniyah, Sulaymaniyah, Iraq \\ ${ }^{4}$ Thalassemia and Congenital Blood Disorders Center, Sulaymaniyah 46001, Iraq \\ Correspondence should be addressed to Sana Dlawar Jalal; dr.sanajalal612@gmail.com
}

Received 18 June 2020; Accepted 19 June 2020; Published 30 June 2020

Copyright (c) 2020 Shaema Salih Amin et al. This is an open access article distributed under the Creative Commons Attribution License, which permits unrestricted use, distribution, and reproduction in any medium, provided the original work is properly cited.

In the article titled "Beta-Thalassemia Intermedia: A Single Thalassemia Center Experience from Northeastern Iraq" [1], there was an error in Table 1. The corrected table is shown below and is listed as Table 1. 
TABle 1: Patient and disease characteristics of the current study.

\begin{tabular}{|c|c|c|c|}
\hline Parameter & Frequency & $\begin{array}{c}\text { Number of } \\
\text { evaluated } \\
\text { patients }\end{array}$ & Percent \\
\hline \multicolumn{4}{|l|}{ Demographic data } \\
\hline \multicolumn{4}{|l|}{ Age (years) } \\
\hline$<18$ & 90 & 159 & 56.6 \\
\hline $18-35$ & 55 & 159 & 34.6 \\
\hline$>35$ & 14 & 159 & 8.8 \\
\hline \multicolumn{4}{|l|}{ Gender } \\
\hline Male & 90 & 159 & 56.6 \\
\hline Female & 69 & 159 & 43.4 \\
\hline Splenectomized & 52 & 159 & 32.75 \\
\hline \multicolumn{4}{|l|}{ Serum ferritin $(\mu \mathrm{g} / \mathrm{L})$} \\
\hline$<1000$ & 122 & 159 & 76.7 \\
\hline$>1000$ & 37 & 159 & 23.3 \\
\hline \multicolumn{4}{|l|}{ Treatment } \\
\hline None transfused & 33 & 159 & 20.7 \\
\hline Occasional transfusion & 75 & 159 & 47.2 \\
\hline Regular transfusion & 51 & 159 & 32.1 \\
\hline Iron chelation & 63 & 159 & 39.6 \\
\hline Hydroxyurea & 75 & 159 & 47.2 \\
\hline \multicolumn{4}{|l|}{ Complications } \\
\hline Facial deformity & 99 & 159 & 62.3 \\
\hline *Osteoporosis & 17 & 60 & 28.3 \\
\hline${ }^{* *}$ Growth retardation & 25 & 90 & 27.8 \\
\hline $\begin{array}{l}{ }^{* * *} \text { Subclinical } \\
\text { hypothyroidism }\end{array}$ & 22 & 131 & 16.8 \\
\hline${ }^{* * * *}$ Cholelithiasis & 19 & 137 & 13.8 \\
\hline Pulmonary hypertension & 18 & 159 & 11.3 \\
\hline $\begin{array}{l}\text { Abnormal liver } \\
\text { function }\end{array}$ & 12 & 159 & 7.5 \\
\hline Thrombosis & 2 & 159 & 1.3 \\
\hline $\mathrm{EMH}$ & 1 & 159 & 0.6 \\
\hline Leg ulcer & 1 & 159 & 0.6 \\
\hline
\end{tabular}

${ }^{*}$ Osteoporosis and ${ }^{* * *}$ subclinical hypothyroidism were evaluated in patients $\geq 10$ years old and/or symptomatic, ${ }^{* *}$ growth retardation (height $>2$ SD below 3rd percentile for the mean age and gender) in patients $\leq 18$ years, and ${ }^{* * * *}$ cholelithiasis estimated in 137 patient excluding the 22 patients that underwent cholecystectomy.

\section{References}

[1] S. S. Amin, S. D. Jalal, K. M. Ali, A. I. Mohammed, L. K. Rasool, and T. J. Osman, "Beta-thalassemia intermedia: a single thalassemia center experience from northeastern Iraq," BioMed Research International, vol. 2020, Article ID 2807120, 11 pages, 2020. 